\title{
A Numerical Solution of the Integral Equation Describing a Photometric Integrating Sphere
}

\author{
Robert L. Brown \\ Institute for Materials Research, National Bureau of Standards, Washington, D.C. 20234
}

(January 10, 1973)

\begin{abstract}
A photometric integrating sphere containing a single disk-shaped baffle and a point source with an arbitrary angular intensity distribution is described by an integral equation. Numerical solutions of this equation are presented for several different source distributions. The errors involved in comparing the various sources are evaluated as a function of baffle size and position.
\end{abstract}

Key words: Integrating sphere; photometry.

\section{Introduction}

The integrating sphere is used in photometry to measure total luminous flux. In a sphere with a diffusely reflecting coating, the illuminance by reflected light is approximately the same at all points on the sphere surface and is proportional to the total flux emitted by the lamp. This reflected light is measured at a point on the sphere surface which is shielded from the direct rays of the lamp by a baffle. Its intensity depends somewhat on the geometry and reflectance of the objects inside the sphere and on the angular intensity distribution of the light from the lamp. Unless this dependence is known, errors will occur in measuring the luminous flux from different types of lamps.

It is possible to describe the nonempty integrating sphere by an integral equation $[1],{ }^{1}$ whose solution gives the illuminance by reflected light at every point on all the surfaces inside the sphere: The present paper describes a numerical solution of this equation for a sphere containing a point source with an arbitrary angular intensity distribution and a disk-shaped baffle located perpendicular to the polar axis of the sphere. The existence of an $\infty$-fold axis of symmetry simplified the work considerably by making it possible to do the azimuthal integrations analytically. The illuminance of the surface inside the sphere was calculated for several values of the baffle radius and its position along the polar axis. Although hemispherical source distributions were used for most of the calculations, the results are applicable to any small lamp whose intensity distribution is known. The extension of the technique to

\footnotetext{
${ }^{1}$ Figures in brackets indicate the literature references on page.
}

larger sources, like fluorescent lamps, should not be difficult. For convenience, uniform reflectance was assumed for all surfaces. The model, however, requires only that the reflection coefficient have axial symmetry about the polar axis. No consideration was given here to the effects of nondiffuse reflection.

\section{Theory}

\subsection{General Integral Equation}

Let $d a$ be an area element of the baffle or inside sphere surface about a point labeled $a$, and let $d a^{\prime}$ be another element of area about a point $a^{\prime}$. Let $\mathbf{x}$ be the vector directed from $a^{\prime}$ to $a$, and let $\mathbf{n}^{\prime}$ and $\mathbf{n}$ be the respective unit outward normals to $d a^{\prime}$ and $d a$. Let $E(a)$ represent the illuminance at point $a$ by reflected light only, and let $E_{0}(a)$ be the illuminance caused by direct light from the source. This latter quantity is determined by the overall geometry and angular intensity distribution of the source. All surfaces are assumed to be perfect diffuse reflectors. Let $\rho(a)$ be the total reflectance at point $a$. The flux $d F\left(a, a^{\prime}\right)$ coming from $d a^{\prime}$ to $d a$ is given by the expression

$$
\begin{aligned}
& d F\left(a, a^{\prime}\right)=\rho\left(a^{\prime}\right)\left[E_{0}\left(a^{\prime}\right)+E\left(a^{\prime}\right)\right] \\
& d a^{\prime}\left(-\mathbf{x} \cdot \mathbf{n}^{\prime}\right) d a(\mathbf{x} \cdot \mathbf{n}) \times S\left(a, a^{\prime}\right) /\left(\pi x^{4}\right) .
\end{aligned}
$$

A derivation of this equation is given by Jacquez and Kuppenheim [2]. In the present work a factor $S\left(a, a^{\prime}\right)$ has been introduced to account for the screening of one part of the sphere's surface from another by the baffle. If all points on $d a^{\prime}$ can be seen from $a$, then $S\left(a, a^{\prime}\right)=1$. If $d a^{\prime}$ is completely or partially screened 
from $a$ by the baffle, then $S\left(a, a^{\prime}\right)$ will be zero or some number less than unity. The illuminance $E(a)$ of $d a$ by reflected light is then equal to the integral of the quantity $d F\left(a, a^{\prime}\right) / d a$ over all the area elements $d a^{\prime}$ on the inside of the sphere. This, of course, includes the baffle surface as well as the sphere surface. The unknown quantity $E(a)$ is thus expressed as an integral containing itself and quantities which are known. We have then,

$$
\begin{aligned}
E(a)=\int_{a^{\prime}} \rho\left(a^{\prime}\right) & {\left[E_{0}\left(a^{\prime}\right)\right.} \\
& \left.+E\left(a^{\prime}\right)\right] T\left(a, a^{\prime}\right) S\left(a, a^{\prime}\right) d a^{\prime} / \pi
\end{aligned}
$$

where

$$
T\left(a, a^{\prime}\right) \equiv\left(-\mathbf{x} \cdot \mathbf{n}^{\prime}\right)(\mathbf{x} \cdot \mathbf{n}) / x^{4} .
$$

The numerical solution of (2) can be obtained by first dividing the surfaces involved into a number of small area elements $\delta a^{\prime}$ having roughly the same size, and approximating the integral by a sum over these $\delta a^{\prime}$. Values of the functions $S\left(a, a^{\prime}\right)$ and $T\left(a, a^{\prime}\right)$ for all possible pairs of area elements are fixed by the geometry of the system, while $\rho(a)$ and $E_{0}(a)$ are assumed to be specified. One then guesses a solution for $E(a)$, say $E^{0}(a)$, and uses (2) to get a first approximation $E^{1}(a)$. The process is repeated using this first approximation in (2). Repetition of this procedure gives a sequence of functions $E^{i}(a)$ which may converge. If it does, the accuracy of the solution may be checked by repeating the whole calculation with smaller area elements $\delta a^{\prime}$, or with a better approximation to the integral, to see whether the limiting value of the function $E(a)$ changes significantly. The former method was used in the present work.

Equation (2) is quite general except for the restriction that the surfaces be diffuse reflectors. In practice, the difficulties involved in evaluating $S\left(a, a^{\prime}\right)$ and $T\left(a, a^{\prime}\right)$ make it desirable to keep the geometry as simple as possible. This will also reduce the number of terms needed in the representation of (2) as a sum, thereby saving time on the iterative calculations. In particular, the use of an experimental arrangement having an $\infty$-fold $\left(C_{\infty}\right)$ axis of symmetry greatly simplifies all aspects of the analysis. Furthermore, this symmetry restriction does not impose any symmetry requirements on the angular intensity distribution of the source. It need be applied only to the geometry and reflectance of the surfaces in the sphere.

\subsection{Specific Model Used in the Calculation}

$$
\text { a. Classification of Surfaces }
$$

The sphere geometry in the present calculation was chosen to approximate the substitution method of using the sphere. Positions of the various surface elements were specified in spherical coordinates with the origin at the sphere center. This model had the following characteristics: (a) A point source at the center of the sphere of radius $R$, with no restrictions on its angular intensity distribution.

(b) A flat, infinitely thin, circular baffle of radius $B$ with its center on the polar axis a distance $D$ from the sphere center and oriented normal to the polar axis.

(c) Uniform reflectance for sphere and baffle surfaces.

For this model, the solution of (2) requires the evaluation of five integrals. Let the symbol $s$ designate points on the sphere, $a_{N}$ points on the north side of the baffle, and $a_{S}$ points on the south side. Let $d E=d E\left(a, a^{\prime}\right)$ be the illuminance at point $a$ produced by the area element $d a^{\prime}$ around $a^{\prime}$. Equation (2) then takes the form

$$
\begin{aligned}
E(s) & =\int_{s^{\prime}} d E\left(s, s^{\prime}\right)+\int_{a_{N^{\prime}}} d E\left(s, a_{N^{\prime}}\right)+\int_{a_{S^{\prime}}} d E\left(s, a_{S^{\prime}}\right) \\
E\left(a_{N}\right) & =\int_{s^{\prime}} d E\left(a_{N}, s^{\prime}\right) \\
E\left(a_{S}\right) & =\int_{s^{\prime}} d E\left(a_{S}, s^{\prime}\right) .
\end{aligned}
$$

Numerical integration of (4) is necessary only for the polar angle $\theta$ since the integration over the azimuthal angle $\varphi$ can be done analytically. Throughout the discussion, points on the sphere surface will be specified by the coordinates $\theta$ and $\varphi$ written without subscripts. The radial coordinate $r$ is a constant equal to $R$ for these points. Baffle points will be designated by the subscript $B$ on the spherical coordinates. The radial coordinate $r_{B}$ is related to $\theta_{B}$ by the equation

$$
r_{B}=D \cos \theta_{B} .
$$

\section{b. Separation of the Azimuthal and Polar Integrations}

When both $a$ and $a^{\prime}$ are on the sphere, $T\left(a, a^{\prime}\right)$ is a constant. If one of the points is on the baffle, the determination of $S\left(a, a^{\prime}\right)$ is trivial. To carry out the integration involving the azimuthal variable it is therefore only necessary to calculate the azimuthal dependence of $S\left(a, a^{\prime}\right)$ when both points are on the sphere, and that of $T\left(a, a^{\prime}\right)$ when one point is on the baffle. These calculations are outlined in appendices I and II. Following this, $E_{0}(a)$ for the different surfaces must be calculated in terms of the angular intensity distribution of the source. This is done in appendix III. For convenience, it will be assumed that the source intensity distribution is independent of the azimuthal angle $\varphi$. This means that $E(a)$ will also be independent of $\varphi$. It is important to note, however, that the value of $E(a)$ at a pole of the sphere depends only on the values of $E_{0}(a)$ averaged over $\varphi$, provided the reflection coefficient has $C_{\infty}$ symmetry. If the observation point is at a pole there are no restrictions on the azimuthal variations in the source intensity. This point will be discussed in appendix IV.

\section{c. Method of Integrating Equation (2) over the Polar Angle $\theta$}

Because of the $C_{\infty}$ symmetry of the sphere and baffle, it was possible to perform the integration of (2) over 
the azimuthal angle $\varphi$ analytically. This integration is discussed in appendices II and III. After this integration the five integrals in (4) become

$$
\begin{aligned}
\int_{s^{\prime}} d E\left(s, s^{\prime}\right) & =\int_{0}^{\pi} \rho\left(\theta^{\prime}\right)\left[E_{0}\left(\theta^{\prime}\right)\right. \\
& \left.+E\left(\theta^{\prime}\right)\right] \sigma\left(\theta, \theta^{\prime}\right) \sin \theta^{\prime} d \theta^{\prime} /(4 \pi)
\end{aligned}
$$$$
\int_{a_{N^{\prime}}} d E\left(s, a_{N^{\prime}}\right)=\int_{0}^{\theta_{s}} \rho_{N}\left(\theta_{B^{\prime}}\right) E_{N}\left(\theta_{B^{\prime}}\right) \tau\left(\theta, \theta_{B^{\prime}}\right) D^{2}
$$$$
\cos \theta_{B^{\prime}} \sin \theta_{B^{\prime}} d \theta_{B^{\prime}} / \pi, \quad \theta<\theta_{e}
$$

$\int_{a_{S^{\prime}}} d E\left(s, a_{S^{\prime}}\right)=\int_{0}^{\theta_{s}} \rho_{S}\left(\theta_{B^{\prime}}\right)\left[E_{0}\left(\theta_{B^{\prime}}\right)+E_{S}\left(\theta_{B^{\prime}}\right)\right]$

$$
\begin{aligned}
\tau\left(\theta, \theta_{B^{\prime}}\right) \times D^{2} \cos \theta_{B^{\prime}} \sin \theta_{B^{\prime}} d \theta_{B^{\prime}} / \pi, \quad \theta>\theta_{e} \\
\int_{s^{\prime}} d E\left(a_{N}, s^{\prime}\right)=\int_{0}^{\theta_{e}} \rho\left(\theta^{\prime}\right)\left[E_{0}\left(\theta^{\prime}\right)\right. \\
\left.+E\left(\theta^{\prime}\right)\right] \tau\left(\theta^{\prime}, \theta_{B}\right) R^{2} \sin \theta^{\prime} d \theta^{\prime} / \pi
\end{aligned}
$$$$
\int_{s^{\prime}} d E\left(a_{S}, s^{\prime}\right)=\int_{\theta_{e}}^{\pi} \rho\left(\theta^{\prime}\right)\left[E_{0}\left(\theta^{\prime}\right)\right.
$$$$
\left.+E\left(\theta^{\prime}\right)\right] \tau\left(\theta^{\prime}, \theta_{B}\right) R^{2} \sin \theta^{\prime} d_{\theta^{\prime}} / \pi .
$$

The functions $E_{0}(\theta), E_{0}\left(\theta_{B}\right), \sigma\left(\theta, \theta^{\prime}\right)$, and $\tau\left(\theta, \theta_{B}\right)$ have been defined by (A7), (A8), (A3), and (A5), respectively. Reflection coefficients are designated by $\rho(\theta), \rho_{N}(\theta)$, and $\rho_{S}(\theta)$ for sphere points, north baffle points, and south baffle points. The functions $E(\theta), E_{N}\left(\theta_{B}\right)$, and $E_{S}\left(\theta_{B}\right)$ are averages over the azimuthal angle of the indirect illuminance for the sphere and baffle points. The parameters $\theta_{s}$ and $\theta_{e}$ are discussed below. The next step, integration over the polar angle, was done numerically, by a modified form of the trapazoidal rule.

To do this, the range covered by $\theta$ was divided into 20 (or 40) equal intervals $\delta \theta$. These are shown in figure 1 along with the other sphere and baffle parameters, $D, B, R, \theta_{s}, \theta_{e}$, and $\theta_{i}$. The angle $\theta_{s}$ has the value $\tan ^{-1}(B / D)$; it defines the portion of the sphere shielded from the direct rays of the source. Thus, we have $E_{0}(\theta)=0$ for $\theta<\theta_{s}$. The angle $\theta_{e}$ is determined by the intersection of the plane of the baffle with the surface of the sphere. It has the value $\cos ^{-1}$ $(D / R)$. Sphere points for which $\theta<\theta_{e}$ cannot be seen from the south side of the baffle, while those for which $\theta>\theta_{e}$ cannot be seen from the north side. We have adopted the restriction that $\theta_{e}$ must equal a value of $\theta$ which marks a boundary between two $\delta \theta$ intervals (see figure 1). This in turn limits the number of $D$ values that may be used. This is an unnecessary restriction which could be relaxed. The angles $\theta_{i}$ are defined by the equation

$$
\theta_{i}=\left(i-\frac{1}{2}\right) \delta \theta \quad i=1 \ldots 20(\text { or } 40) .
$$

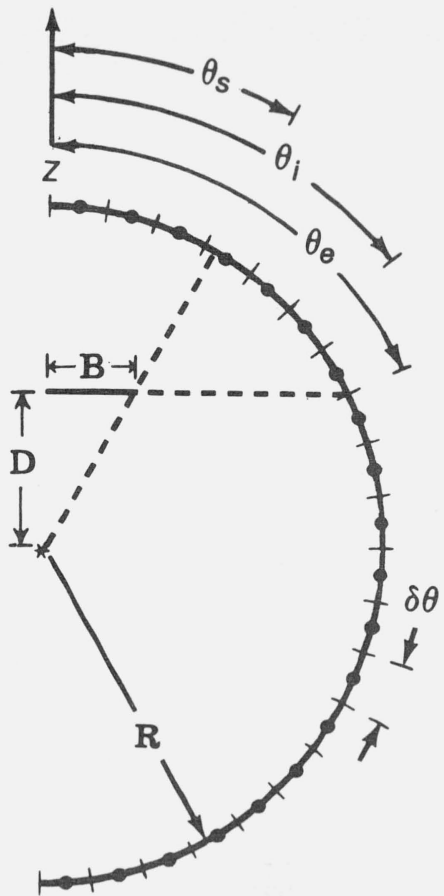

FIGURE 1. Definition of sphere parameters.

$R$ is the radius of the sphere; $B$ is the baffle radius, and $D$ its distance from the center of the sphere where the light source is located. The positive $z$ axis passes through the north pole. The range of the polar angle $\theta$ is divided into 20 equal intervals $\delta \theta$. Their centers are denoted by the polar angles $\theta_{i}, i=1 \ldots 20 . \theta_{s}$ defines the portion of the sphere shielded from the direct rays of the source. $\theta_{e}$ is determined by the intersection of the plane of the baffle with the surface of the sphere.

They thus lie at the midpoints of the $\delta \theta$ intervals. In figure 1 , the circles on the perimeter of the semicircle indicate the $\theta_{i}$ values, and the other index lines show the boundaries of the $\delta \theta$ intervals.

The range covered by $\theta_{B}$ was not divided into equal intervals. Instead, the baffle radius was divided into 4 equal parts, of length $\delta B=B / 4$. The centers of these intervals fall at distances $B_{i}$ from the center of the baffle. The $B_{i}$ are given by the equation $B_{i}=\left(i-\frac{1}{2}\right) \delta B$, $i=1$. . . 4. The values of $\theta_{B i}$ corresponding to these positions are given by

$$
\theta_{B i}=\tan ^{-1}\left(B_{i} / D\right) \quad i=1 \ldots 4 .
$$

The increment $\delta \theta_{B}$ thus depends on $i$;

$$
\delta \theta_{B i}=\delta B\left(D \cos ^{2} \theta_{B i}\right)^{-1} \quad i=1 \ldots 4 .
$$

Before representing the integrals in (6) by summations, let us examine how rapidly the different functions in the integrals vary over intervals of size $\delta \theta$. A study of figure 1 will show that the functions $E(\theta), E_{N}\left(\theta_{B}\right)$, $E_{S}\left(\theta_{B}\right)$, and $\tau\left(\theta, \theta_{B}\right)$ experience only small relative changes over any of the $\delta \theta$ or $\delta \theta_{B}$ intervals. The functions $E_{o}(\theta), E_{o}\left(\theta_{B}\right)$, and $\sigma\left(\theta, \theta^{\prime}\right)$, however, can change markedly over certain $\delta \theta$ intervals. For instance, $E_{o}(\theta)$ has a discontinuity in the $\delta \theta$ interval which contains $\theta_{s}$. To cope with these rapid variations, the slowly varying functions were assumed to be constant over the interval $\delta \theta$ and were taken out from under the inte- 
gral sign. The remaining portion of the integral was then approximated by dividing $\delta \theta$ into 10 equal parts and summing over these smaller intervals. The same procedure could be used for the intervals $\delta \theta_{B}$ if ever there is a large variation in $E_{0}\left(\theta_{B}\right)$.

Written as a summation, the integral equation (4) has the form

$$
\begin{aligned}
\boldsymbol{E}_{i} & =\sum_{j=1}^{m}\left[(D A A)_{i j}+(A A)_{i j} E_{j}\right] \\
& +\sum_{j=1}^{4}\left[(A N)_{i j} E N_{j}+(D A S)_{i j}\right. \\
& \left.+(A S)_{i j} E S_{j}\right] \quad i=1 \ldots m \\
E N_{i} & =\sum_{j=1}^{e}\left[(D N A)_{i j}+(N A)_{i j} E_{j}\right] \quad i=1 \ldots 4 \\
E S_{i} & =\sum_{j=e+1}^{m}\left[(D S A)_{i j}+(S A)_{i j} E_{j}\right] \quad i=1 \ldots 4
\end{aligned}
$$

where $E_{i}$ is the value of $E(\theta)$ when $\theta=\theta_{i}, E N_{i}$ and $E S_{i}$ are the values of $E_{N}\left(\theta_{B}\right)$ and $E_{S}\left(\theta_{B}\right)$ when $\theta_{B}=\theta_{B i}$, $\boldsymbol{e}=\theta_{e} / \delta \theta, m=20$ or 40 , and the various coefficients are defined as follows:

$$
(A A)_{i j}=\rho\left(\theta_{j}\right) \int_{j-}^{j+} \sigma\left(\theta_{i}, \theta\right) \sin \theta d \theta /(4 \pi)
$$

where $j \pm=\theta_{j} \pm \frac{1}{2} \delta \theta$, and the integral is evaluated numerically;

$$
\begin{aligned}
& (A N)_{i j}=\rho_{N}\left(\theta_{B j}\right) \tau\left(\theta_{i}, \theta_{B j}\right) D^{2} \cos \theta_{B j} \sin \theta_{B j} \delta \theta_{B j} / \pi \\
& i=1 \ldots e \\
& =0 \quad i=e+1 \ldots m \\
& (A S)_{i j}=\rho_{S}\left(\theta_{B j}\right) \tau\left(\theta_{i}, \theta_{B j}\right) D^{2} \cos \theta_{B j} \sin \theta_{B j} \delta \theta_{B j} / \pi \\
& i=e+1 \ldots m \\
& =0 \quad i=1 \ldots e \\
& (N A)_{i j}=\rho\left(\theta_{j}\right) \tau\left(\theta_{j}, \theta_{B i}\right) R^{2} \sin \theta_{j} \delta \theta / \pi \\
& j=1 \ldots e \\
& =0 \quad j=e+1 \ldots m \\
& (S A)_{i j}=\rho\left(\theta_{j}\right) \tau\left(\theta_{j}, \theta_{B i}\right) R^{2} \sin \theta_{j} \delta \theta / \pi \\
& j=e+1 \ldots m \\
& =0 \quad j=1 \ldots e .
\end{aligned}
$$

The coefficient $(D A A)_{i j}$ is given by (11) with the addition of $E_{0}(\theta)$ under the integral sign. We can write $(D A S)_{i j}=E_{o}\left(\theta_{B j}\right)(A S)_{i j}$ unless $E_{o}\left(\theta_{B}\right)$ shows appreci- able variation over $\delta \theta_{B j}$, in which case $(D A S)_{i j}$ should be written as an integral over $\delta \theta_{B j}$. The remaining coefficients $(D N A)_{i j}$ and $(D S A)_{i j}$ are equivalent to the expressions (14) and (15), each multiplied by $E_{o}(\theta)$ and integrated over the range $j \pm$ as in (11).

\subsection{Solution of the Integral Equation}

As outlined in (IIA), an iterative method was used to solve (10), which is the integral equation (2) approximated by a summation. Equation (10) contains the unknown quantities $E_{i}, E N_{i}$, and $E S_{i}$ and a series of coefficients which depend on the geometry and reflectance of the surfaces, and on the angular intensity distribution of the source.

A convenient zeroth order approximation is the solution of (2) for a sphere containing only a point source with a uniform intensity distribution. For area elements on a spherical surface, $T\left(a, a^{\prime}\right)=1 /\left(4 R^{2}\right)$ for all $a, a^{\prime}$. Without the baffle, we also have $S\left(a, a^{\prime}\right)$ $=1$ for all $a, a^{\prime}$. The integral (2) in this case becomes

$$
E(a)=\int_{a^{\prime}} \rho\left(a^{\prime}\right)\left[E_{0}\left(a^{\prime}\right)+E\left(a^{\prime}\right)\right] d a^{\prime} /\left(4 \pi R^{2}\right)
$$

where the integration is over the sphere surface. Since this integral contains nothing which depends on $a$, we must have $E(a)$ independent of position. Letting $E(a)=E$, we can solve (16) for $E$ to get

$$
E=\int_{a^{\prime}} \rho\left(a^{\prime}\right) E_{0}\left(a^{\prime}\right) d a^{\prime}\left[1-\int_{a^{\prime}} \rho\left(a^{\prime}\right) d a^{\prime} /\left(4 \pi R^{2}\right)\right]^{-1}
$$

$$
\left(4 \pi R^{2}\right)^{-1}
$$

For uniform reflectance $\rho$, and a centrally placed source with a uniform angular intensity distribution, one has $E_{0}(a)=E_{0}$, and (17) becomes

$$
E=\rho E_{0} /(1-\rho) .
$$

The value $E$ calculated from this equation was used as the first approximation to all the unknowns, $E_{i}$, $E N_{i}$, and $E S_{i}$.

With $\rho=0.9$, values of $\boldsymbol{E}(a)$ satisfied (10) to better than 1 part in $10^{5}$ after about 35 iterations on a high speed computer for both the 24 and 44 point models. The solutions were checked to see that they satisfied the condition of flux balance. This is given by the relation

$$
\int_{a} E_{0}(a) d a=\int_{a}[1-\rho(a)]\left[E_{0}(a)+E(a)\right] d a
$$

where the integrations are over all the surfaces in the sphere. The integral on the left is the total flux into the sphere, and that on the right, the total flux out. 


\section{Calculations and Results}

\subsection{Description of Source Distributions}

Two source intensity distributions, each uniform in one hemisphere and zero in the other, were used for most of the calculations. Specifically, these distributions were

$$
\begin{aligned}
I_{N}(\theta) & \equiv 2 \mathrm{~lm} / \mathrm{sr} & & 0 \leqq \theta<\pi / 2 \\
& \equiv 0 & & \pi / 2 \leqq \theta \leqq \pi \\
I_{S}(\theta) & \equiv 0 & & 0 \leqq \theta<\pi / 2 \\
& \equiv 2 & & \pi / 2 \leqq \theta \leqq \pi .
\end{aligned}
$$

The total flux $F_{0}$ in each case is, by (A10), equal to $4 \pi \mathrm{lm}$.

With the sphere geometry discussed in the next section, an additional group of distributions having the form

$$
I_{i}(\theta) \equiv\left[\sin \theta_{i} \sin \left(\frac{1}{2} \delta \theta\right)\right]^{-1} \mathrm{~lm} / \mathrm{sr}
$$

$$
\theta_{i}-\frac{1}{2} \delta \theta<\theta<\theta_{i}+\frac{1}{2} \delta \theta
$$

$$
\equiv 0 \quad \text { (all other } \theta \text { values) }
$$

was used. The $\theta_{i}$ are given by (7). The total flux is the same for each distribution and is equal to $4 \pi \mathrm{lm}$. We shall discuss later how the results obtained from this series of distributions can be used to determine $E(a)$ for an arbitrary source distribution.

\subsection{Results for a Reference Sphere}

For much of the work, a particular set of sphere parameters was used. They were chosen to correspond roughly to those of a typical experimental sphere. They were, $R=1, B=0.1$, and $D=0.31 \mathrm{~m}$. A uniform reflection coefficient of 0.9 was used for all surfaces. Calculated values of the indirect illuminance $E(\theta)$ for the 24-point model are shown as polar plots in figures 2 and 3 for the north and south hemispherical source distributions, respectively. If there were no baffle, $E(\theta)$ would have a uniform value of $9 \mathrm{~lm} / \mathrm{m}^{2}$. The $E(\theta)$ values shown in figures 2 and 3 are lower than this on the average because of light absorption by the baffle. The $E(\theta)$ values are enhanced on the side of the sphere opposite that being directly illuminated. Values of the indirect illuminance of the baffle show the same effect. They are shown in table 1 for the reference sphere and the hemispherical distributions. For the north distribution, the north side of the baffle, being opposite the directly illuminated surfaces, receives more indirect illumination than the south side. The opposite situation holds for the south distribution.

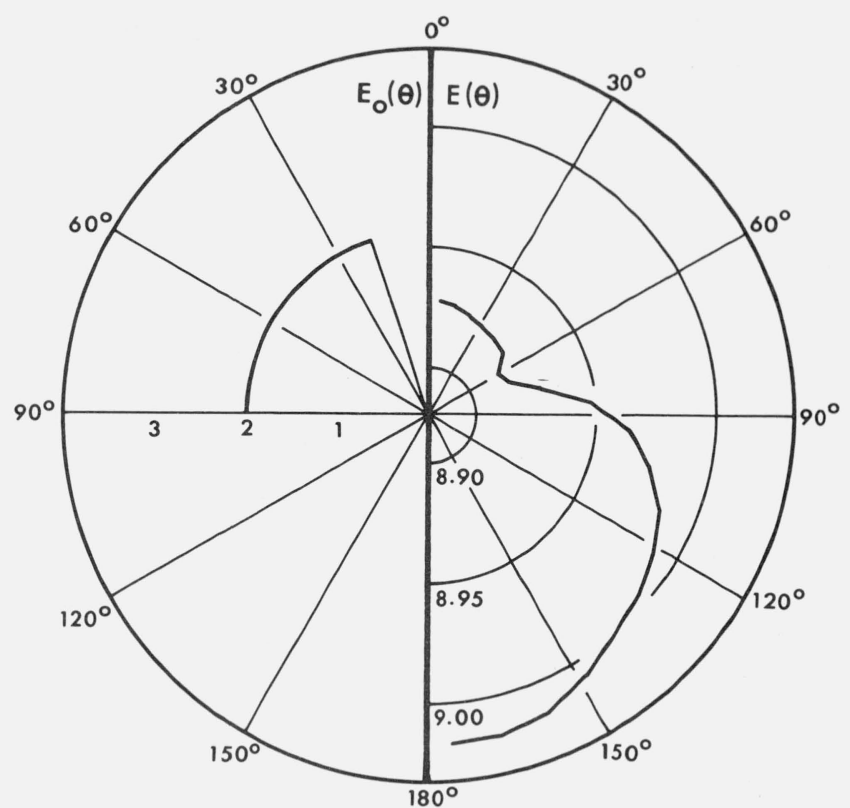

FIGURE 2. Variation with the polar angle of the indirect illuminance $\mathrm{E}(\theta)$ for the northward directed hemispherical source distribution, and the reference sphere geometry.

$E_{0}(\theta)$ is the direct illuminance.

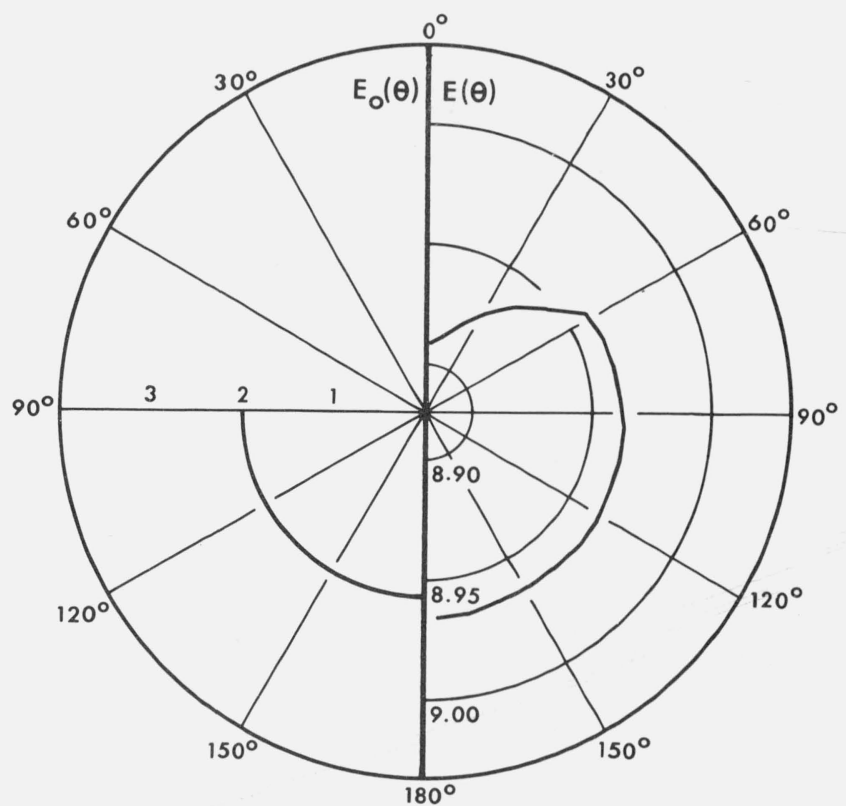

FIGURE 3. Variation with the polar angle of the indirect illuminance $\mathrm{E}(\theta)$ for the southward directed hemispherical source distribution, and the reference sphere geometry. 
TABLE 1. Indirect illuminance of baffle for reference sphere dimensions, $\rho=0.9$, hemispherical source distributions, and 24-point model

\begin{tabular}{c|c|c|c|c|c}
\hline \hline \multicolumn{5}{c|}{$I_{N}(\theta)(\mathrm{lm} / \mathrm{sr})$} & \multicolumn{2}{c}{$I_{S}(\theta)(\mathrm{lm} / \mathrm{sr})$} \\
\hline$i$ & $\theta_{B i}(\mathrm{deg})$ & $E N_{i}\left(\mathrm{~lm} / \mathrm{m}^{2}\right)$ & $E S_{i}\left(\mathrm{~lm}_{/} \mathrm{m}^{2}\right)$ & $E N_{i}\left(1 \mathrm{~m} / \mathrm{m}^{2}\right)$ & $E S_{i}\left(\mathrm{~lm} / \mathrm{m}^{2}\right)$ \\
\hline 1 & 2.32 & 9.5510 & 8.2733 & 8.0889 & 9.7416 \\
2 & 6.94 & 9.5522 & 8.2736 & 8.0888 & 9.7413 \\
3 & 11.46 & 9.5547 & 8.2743 & 8.0886 & 9.7407 \\
4 & 15.86 & 9.5583 & 8.2754 & 8.0883 & 9.7398 \\
\hline
\end{tabular}

This table also shows the large difference in indirect illuminance of the north and south sides of the baffle, and its large dependence on the source distribution. Actually, it is the large change in $E N_{i}$ which is primarily responsible for the change in $E_{1} \approx E(\theta=0)$ (see figs. 2 and 3 ) when the distribution shifts from one hemisphere to the other. The changes in the amount of light coming to the $\theta_{1}$ position from the other surfaces are small by comparison.

Because the detector would normally be at the north pole, it is the change in $E_{1}$ that is of primary interest. For the reference sphere, $E_{1}$ changes by 0.25 percent when the hemispherical source distribution is switched from north to south; values of $E(\theta)$ at other $\theta$ values change to a greater or lesser degree.

\subsection{Effect of Changing the Baffle Position}

The effect of changing the position of the baffle along the polar axis is shown in figure 4. Here, values of $E(\theta)$ are plotted as a function of $\theta$ for four different baffle positions $D$, including that occurring in the reference sphere. The radius of the baffle was held constant at $B=0.1 \mathrm{~m}$. For each case, there are two curves corresponding to the two hemispherical distributions. That arising from the north distribution is labeled $N$. Of the four positions, the difference in $E_{1}$ between the two source distributions is smallest for the baffle position used in the reference sphere. This position does not, however, minimize this difference. A plot of $E_{1}(N)-E_{1}(S)$ versus $D$ at constant $B$, where $N$ and $S$ designate the distributions, is shown in figure 5 by the filled-in circles. This plot shows that for $D \approx 0.28 \mathrm{~m}$ there would be no difference at all in $E_{1}$ between the two distributions. This optimum baffle position differs considerably from the value of $D=$ $0.372 \mathrm{R}$, which minimizes the screened areas of the sphere surface [3]. Minimizing the screened areas does, however, minimize the change in $E(\theta)$ averaged over the sphere that occurs when the hemispherical source is directed from north to south. The sum of the squares of the differences $E_{i}(N)-E_{i}(S)$, each weighted by $\sin \theta_{i}$ to account for the different sizes of the sphere areas represented by the $\theta_{i}$, was smaller for $D=0.38$ than for $D=0.31,0.45$, or $0.28 \mathrm{~m}$. (Values of $E_{i}(N)$ and $E_{i}(S)$ for $D=0.28$ and $0.38 \mathrm{~m}$ were estimated by interpolation between the curves shown in fig. 4.) Thus, while the dependence of the $E(\theta)$ values around the sphere on the source distribution is minimized by $D=0.372$, the experimentally important quantity

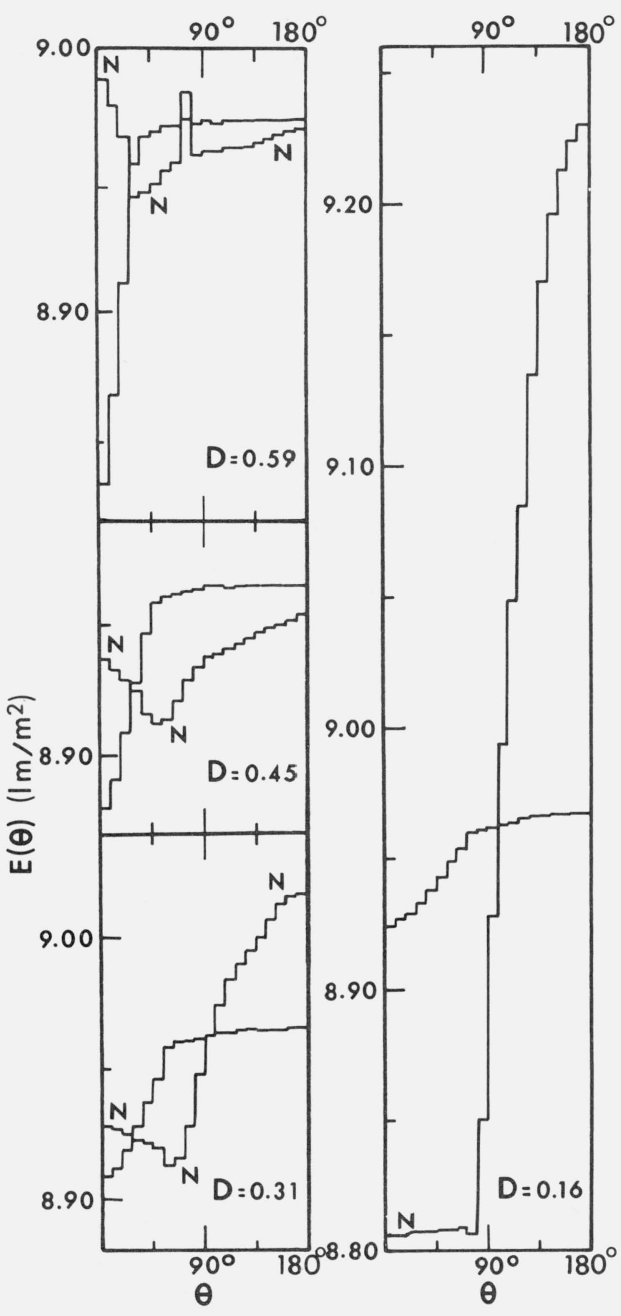

FIGURE 4. Indirect illuminance $\mathrm{E}(\theta)$ versus $\theta$ arising from the north (labeled $\mathrm{N}$ ) and south hemispherical distributions for different values of the baffle position.

The other parameters have reference sphere values.

$E_{1}(N)-E_{1}(S)$ is minimized by $D=0.28 \mathrm{~m}$. At this stage in the calculations it is not possible to generalize this result to other source distributions. The optimum baffle position may not be the same for source distributions differing from the hemispherical ones considered here.

\subsection{Effect of Changing the Baffle Radius}

At a fixed value of $D$, decreasing the baffle radius will decrease the error $E_{1}(N)-E_{1}(S)$ made in observing the hemispherical distributions. Examples of this are shown in figure 5. The three points connected by the dotted line correspond to combinations of $B$ and $D$ which keep constant the area of the sphere screened by the baffle from the observation port. 


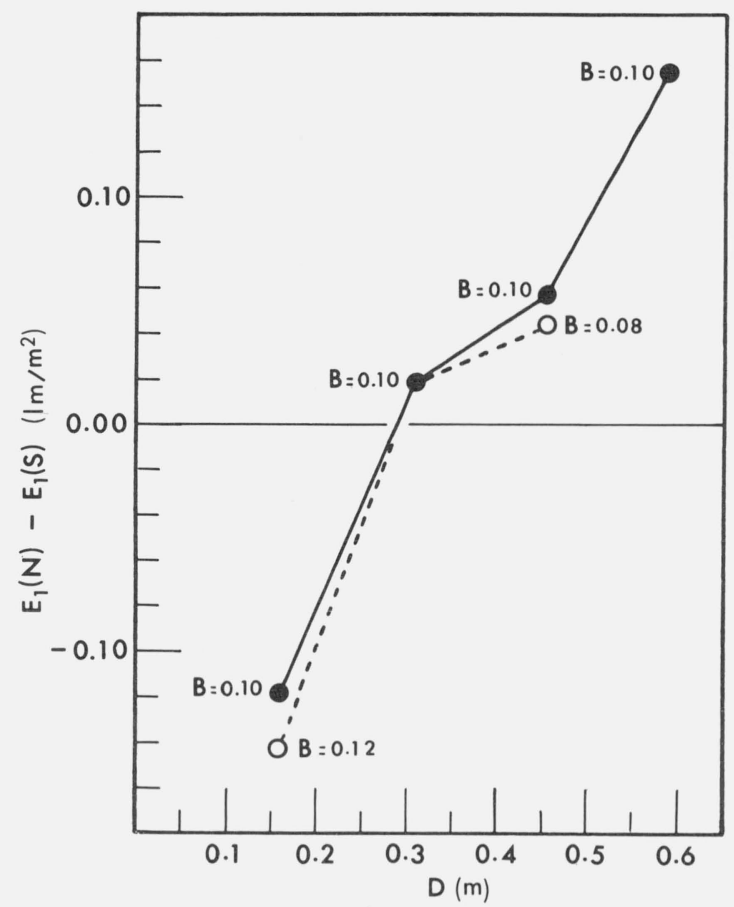

FIGURE 5. Difference $\mathrm{E}_{1}(\mathrm{~N})-\mathrm{E}_{1}(\mathrm{~S})$ between the indirect illuminances near the north pole produced by the north and south hemispherical distributions as a function of baffle radius and position.

\subsection{Determination of $E(\theta \approx 0)$ for an Arbitrary Source Intensity Distribution I( $\theta)$}

Calculations of $E_{1}$ using the reference geometry with the 24-point model were done for a number of the distributions $I_{i}(\theta)$ defined by (20). Several of the $E_{1}$ values were obtained from the others by graphical interpolation. All are shown in table 2.

Consider a source with an intensity distribution $I(\theta)$ which can be approximated by a linear combination of the $I_{i}(\theta)$ distributions. Let

$$
I(\theta)=\sum_{i=1}^{20} w_{i} I_{i}(\theta)
$$

where the weights $w_{i}$ satisfy the relation

$$
\sum_{i=1}^{20} w_{i}=1
$$

Then, if we define $E_{1}(i)$ as the value of $E_{1}$ calculated for the distribution $I_{i}(\theta)$ we have simply

$$
E_{1}=\sum_{i=1}^{20} w_{i} E_{1}(i)
$$

where $E_{1}$ is the value for the distribution $I(\theta)$. Values of $E_{1}(i)$ from table 2 were used with appropriate weights to calculate $E_{1}$ for the hemispherical distributions $I_{N}(\theta)$ and $I_{S}(\theta)$. The results differed by 0.03 percent from those calculated directly from $I_{N}(\theta)$ and $I_{S}(\theta)$. The use of interpolated values for some of the $E_{1}(i)$ is probably responsible for most of the difference.

TABLE 2. Indirect illuminance of north pole region for reference sphere dimensions, $\rho=0.9$, source distributions $\mathrm{I}_{\mathrm{i}}(\theta)$, and 24-point model

\begin{tabular}{r|r|c|c}
\hline \hline$i$ & $\begin{array}{c}\theta_{i} \\
(\mathrm{deg})\end{array}$ & $\begin{array}{c}I_{i}(\theta) \\
(\mathrm{lm} / \mathrm{sr})\end{array}$ & $\begin{array}{c}E_{1} \\
\left(\mathrm{~lm} / \mathrm{m}^{2}\right)\end{array}$ \\
\hline 1 & 4.5 & 159.4 & 7.8329 \\
2 & 13.5 & 54.65 & 8.1143 \\
3 & 22.5 & 33.33 & 9.0464 \\
4 & 31.5 & 24.41 & 9.0216 \\
5 & 40.5 & 19.64 & 9.0010 \\
& & & \\
6 & 49.5 & 16.77 & 8.9823 \\
7 & 58.5 & 14.96 & 8.9666 \\
8 & 67.5 & 13.81 & 8.9542 \\
9 & 76.5 & 13.12 & $\mathrm{a} .951$ \\
10 & 85.5 & 12.79 & a 8.950 \\
& & 12.79 & 8.9500 \\
11 & 94.5 & 13.12 & a 8.950 \\
12 & 103.5 & 13.81 & a 8.949 \\
13 & 112.5 & 14.96 & 8.9487 \\
14 & 121.5 & 16.77 & a 8.947 \\
15 & 130.5 & & a 8.944 \\
16 & 139.5 & 19.64 & 8.9387 \\
17 & 148.5 & 24.41 & 8.8056 \\
18 & 157.5 & 33.33 & 8.4642 \\
19 & 166.5 & 54.65 & 8.0655 \\
20 & 175.5 & 159.4 & \\
\hline
\end{tabular}

a These values were obtained by graphical interpolation between the unmarked values.

\section{Discussion}

The integral equation description of the integrating sphere offers an accurate way to determine the errors caused by the screening effects of objects inside the sphere. While its numerical solution is possible, in principle, for any sphere geometry, a great simplification results when an $\infty$-fold axis of symmetry exists for the various surfaces and their reflection coefficients. The computation time is so short for such a system, that it becomes feasible to improve the accuracy by using smaller area elements and to extend the calculations to spheres containing several objects.

The effect of increasing the number of sphere points from 20 to 40 is shown in table 3 for the reference sphere dimensions. The biggest change in the $E_{i}$ values occurred for $E_{1}$. There were decreases in $E_{1}(N)$ and $E_{1}(S)$ of around 0.03 percent and an increase in their difference $E_{1}(N)-E_{1}(S)$ of 3.1 percent. Further improvements in the accuracy of the calculation could be achieved by using more points, and/or by using a better approximation to the integral.

An extension of the method to spherical and cylindrical sources located anywhere along the sphere axis appears feasible. This would greatly increase the number of systems to which the method could be applied. 
TABLE 3. Effect on indirect illuminance near ${ }^{\mathrm{a}}$ the north pole of decreasing the interval size $\delta \theta$, for reference sphere dimensions, $\rho=0.9$, and hemispherical source distributions

\begin{tabular}{c|l|l|c}
\hline \hline & 20 sphere points & 40 sphere points & $\begin{array}{c}\text { Change } \\
\text { (percent) }\end{array}$ \\
\hline $\mathrm{E}_{1}(N)$ & $8.9277 \mathrm{~lm} / \mathrm{m}^{2}$ & $8.9259 \mathrm{~lm} / \mathrm{m}^{2}$ & 0.020 \\
$E_{1}(S)$ & 8.9087 & 8.9063 & 0.027 \\
$E_{1}(N)-\mathrm{E}_{1}(S)$ & 0.0190 & 0.0196 & -3.1 \\
\hline \hline
\end{tabular}

a The $E(\theta)$ values given here are those at $\theta=4.5^{\circ}$.

b The values at $\theta=4.5^{\circ}$ for the 40 sphere point case were obtained by linear interpolation between the $E(\theta)$ values calculated at $\theta=2.25$ and $6.75^{\circ}$.

The writer thanks S. R. Kraft for suggesting the integral equation approach to the baffle problem and for guidance in the initial phase of the work. Acknowledgment is also given to W. B. Fussel for many valuable discussions.

\section{References}

[1] Moon, P., J. Opt. Soc. Am. 30, 195 (1940).

[2] Jacquez, J. A., Kuppenheim, H. F., J. Opt. Soc. Am. 45, 460 (1955).

[3] Rosa, E. B., and Taylor, A. H., Sci. Papers Bur. Standards No. 447, p. 281 (1922).

(Paper 77A3-773)

\section{Appendix I: Azimuthal dependence of $S\left(a, a^{\prime}\right)$ when both $a$ and $a^{\prime}$ are on the Sphere Surface}

Consider two points $(\theta, \varphi)$ and $\left(\theta^{\prime}, \varphi^{\prime}\right)$ on the surface of the sphere. These points determine a line which intersects the plane containing the baffle at some point. Let $\beta$ be the distance in the plane of the baffle between this point of intersection and the polar axis. Because of the $C_{\infty}$ symmetry of the system, the azimuthal dependence of $\beta$ can be specified in terms of the difference $\varphi-\varphi^{\prime}$. One can thus let $\varphi=0$ and calculate $\beta$ as a function of the three parameters $\theta, \theta^{\prime}$, and $\varphi^{\prime}$. It can be shown that

where

$$
\beta^{2}=f+g \cos \varphi^{\prime}
$$

$$
\begin{aligned}
& f=\frac{1}{4} R^{2}\left[(1-\Gamma)^{2} \sin ^{2} \theta+(1+\Gamma)^{2} \sin ^{2} \theta^{\prime}\right] \\
& g=\frac{1}{2} R^{2}(1-\Gamma)(1+\Gamma) \sin \theta \sin \theta^{\prime} \\
& \Gamma=\left[2 D-R\left(\cos \theta+\cos \theta^{\prime}\right)\right]\left[R\left(\cos \theta^{\prime}-\cos \theta\right)\right]^{-1} .
\end{aligned}
$$

The line between $(\theta, \varphi)$ and $\left(\theta^{\prime}, \varphi^{\prime}\right)$ will intersect the baffle under the conditions, $0 \leqq \theta<\theta_{e}<\theta^{\prime} \leqq \pi$, and $\beta^{2} \leqq B^{2}$, where $\theta_{e}$ is the polar angle determined by the intersection of the baffle plane with the surface of the sphere (see fig. 1). Let $\varphi_{s}^{\prime}$ be that value of $\varphi^{\prime}$ for which $\beta^{2}=B^{2}$. From (Al) we have

$$
\varphi_{s}^{\prime}=\cos ^{-1}\left[\left(B^{2}-f\right) / g\right] .
$$

The azimuthal dependence of $S\left(\theta ; \theta^{\prime}, \varphi^{\prime}\right)$ is thus

$S\left(\theta ; \theta^{\prime}, \varphi^{\prime}\right)=1$ for $0 \leqq \varphi^{\prime} \leqq \varphi_{s}^{\prime}$ and

$$
\begin{aligned}
& \qquad 2 \pi-\varphi_{s}^{\prime} \leqq \varphi^{\prime} \leqq 2 \pi \\
& =0 \text { for } \varphi_{s}^{\prime}<\varphi^{\prime}<2 \pi-\varphi_{s}^{\prime} \\
& =0 \text { for } 0 \leqq \theta<\theta_{e}<\theta^{\prime} \leqq \pi .
\end{aligned}
$$

For the integral over $\varphi^{\prime}$ we have

$$
\sigma\left(\theta, \theta^{\prime}\right) \equiv \int_{0}^{2 \pi} S\left(\theta ; \theta^{\prime}, \varphi^{\prime}\right) d \varphi^{\prime}=2 \varphi_{s}^{\prime} .
$$

The azimuthal integration in the evaluation of the first integral $\int_{s^{\prime}} d E\left(s, s^{\prime}\right)$ in (6) is thus accomplished since $S\left(a, a^{\prime}\right)$ is the only factor in the integrand which contains $\varphi^{\prime}$.

\section{Appendix II: Azimuthal Dependence of $T\left(a, a^{\prime}\right)$ When One Point Is on the Baffle and the Other Is on the Sphere}

Consider a point $(\theta, \varphi)$ on the sphere surface and a point $\left(\theta_{B}, \varphi_{B}\right)$ on the baffle. It can be shown that

$$
\begin{aligned}
T\left(\theta, \varphi ; \theta_{B}, \varphi_{B}\right)=k\left[f_{1}+g_{1} \cos \left(\varphi-\varphi_{B}\right)\right] \\
{\left[f_{2}+g_{2} \cos \left(\varphi-\varphi_{B}\right)\right]^{-2} }
\end{aligned}
$$

where

$$
\begin{aligned}
& k= \pm\left(D \cos ^{2} \theta_{B}-R \cos \theta\right) \\
& f_{1}=R-D \cos ^{2} \theta_{B} \cos \theta \\
& g_{1}=-D \cos \theta_{B} \sin \theta_{B} \sin \theta \\
& f_{2}=R^{2}+D \cos ^{2} \theta_{B}(D-2 R \cos \theta) \\
& g_{2}=-2 R D \cos \theta_{B} \sin \theta_{B} \sin \theta .
\end{aligned}
$$

In the expression for $k$, the negative sign is used for baffle points on the north side, and the positive sign for points on the south side.

As one would expect from the $C_{\infty}$ symmetry, $T(\theta, \varphi$; $\left.\theta_{B}, \varphi_{B}\right)$ depends on the difference $\varphi-\varphi_{B}$ between the azimuthal angles. The integral over either azimuthal angle is

$$
\begin{aligned}
\tau\left(\theta, \theta_{B}\right) & \equiv \int_{0}^{2 \pi} T\left(\theta, \varphi ; \theta_{B}, \varphi_{B}\right) d \varphi \\
& =\int_{0}^{2 \pi} T\left(\theta, \varphi ; \theta_{B}, \varphi_{B}\right) d \varphi_{B} \\
& =2 \pi k\left(f_{1} f_{2}-g_{1} g_{2}\right)\left(f_{2}^{2}-g_{2}^{2}\right)^{-3 / 2}
\end{aligned}
$$


This function $\tau\left(\theta, \theta_{B}\right)$ furnishes the azimuthal portion of all the integrals in (4) except the first.

\section{Appendix III: Determination of the Direct Illuminance $E_{0}(a)$}

Let $I(\theta, \varphi)$ be the angular intensity distribution of the point source in lumen/steradian. The direct illuminance $E_{0}(\theta, \varphi, r)$ at a point $(\theta, \varphi, r)$ is given by the expression

$$
E_{0}(\theta, \varphi, r)=I(\theta, \varphi)(\mathbf{r} \cdot \mathbf{n}) r^{-3}
$$

where $\mathbf{r}$ is the vector from the source (located at the origin) to the point and $\mathbf{n}$ is the unit outward normal from the area element about the point. For sphere points $(\mathbf{r} \cdot \mathbf{n}) r^{-3}$ has the value $1 / R^{2}$, while for baffle points it has the value $1 /\left(D^{2} \cos \theta_{B}\right)$. When $\rho(a)$ has $C_{\infty}$ symmetry, $E(a)$ at the poles is independent of the azimuthal dependence of $I(\theta, \varphi)$. If the observation point is at a pole it is permissible (see appendix IV) to use the average of $E_{0}(\theta, \varphi)$ over $\varphi$ in the calculation.

For points on the sphere surface, excepting those screened from the source by the baffle, we have

$$
\begin{aligned}
E_{0}(\theta) \equiv(1 / 2 \pi) \int_{0}^{2 \pi} E_{0}(\theta, \varphi) d \varphi & \\
& =(1 / 2 \pi) \int_{0}^{2 \pi} I(\theta, \varphi) d \varphi / R^{2} .
\end{aligned}
$$

For baffle points, we have

$$
\begin{aligned}
E_{0}\left(\theta_{B}\right) \equiv(1 / 2 \pi) \int_{0}^{2 \pi} E_{0}\left(\theta_{B}, \varphi_{B}\right) d \varphi_{B} \\
=\left(D^{2} \cos \theta_{B}\right)^{-1}(1 / 2 \pi) \int_{0}^{2 \pi} I\left(\theta_{B}, \varphi_{B}\right) d \varphi_{B} .
\end{aligned}
$$

The north, south designation is not needed here, since $E_{0}\left(\theta_{B}\right)$ is zero on the north side of the baffle. One need only know $I(\theta, \varphi)$ averaged over $\varphi$; i.e.,

$$
I(\theta) \equiv(1 / 2 \pi) \int_{0}^{2 \pi} I(\theta, \varphi) d \varphi
$$

The total flux $F_{0}$ from this source is given by the integral

$$
F_{0}=\int_{4 \pi} I(\theta, \varphi) d \Omega=2 \pi \int_{0}^{\pi} I(\theta) \sin \theta d \theta
$$

where $d \Omega$ is the element of solid angle and the integration is over $4 \pi$ steradians.

\section{Appendix IV: On the Absence of Symmetry Restrictions on the Source Intensity Distribution}

When the reflection coefficient has $C_{\infty}$ symmetry, and the observation point is at the pole, there are no symmetry restrictions on the angular intensity distribution of the source. To see why this is so consider the integral equation (2) for points on the sphere near the north pole.

$$
\begin{aligned}
& E(\theta, \varphi)=\int_{0}^{\pi} \rho\left(\theta^{\prime}\right)\left[\int _ { 0 } ^ { 2 \pi } \left[E_{0}\left(\theta^{\prime}, \varphi^{\prime}\right)\right.\right. \\
& \left.\left.+E\left(\theta^{\prime}, \varphi^{\prime}\right)\right] S\left(\theta, \varphi ; \theta^{\prime}, \varphi^{\prime}\right) d \varphi^{\prime}\right] \times \sin \theta^{\prime} d \theta^{\prime} / 4 \pi \\
& +\int_{0}^{\theta_{s}} \rho_{N}\left(\theta_{B^{\prime}}\right)\left[\int_{0}^{2 \pi} E_{N}\left(\theta_{B^{\prime}}, \varphi_{B^{\prime}}\right) T\left(\theta, \varphi ; \theta^{\prime}, \varphi^{\prime}\right) d \varphi_{B^{\prime}}\right] \\
& \times D^{2} \cos \theta_{B^{\prime}} \sin \theta_{B^{\prime}} d \theta_{B^{\prime}} / \pi
\end{aligned}
$$

The average of $E(\theta, \varphi)$ over $\varphi$ is

$$
\begin{aligned}
E(\theta) & \equiv(1 / 2 \pi) \int_{0}^{2 \pi} E(\theta, \varphi) d \varphi \\
& =\int_{0}^{\pi} \rho\left(\theta^{\prime}\right)\left[E_{0}\left(\theta^{\prime}\right)+E\left(\theta^{\prime}\right)\right] \sigma\left(\theta, \theta^{\prime}\right) \sin \theta^{\prime} d \theta^{\prime} / 4 \pi
\end{aligned}
$$$$
+\int_{0}^{\theta s} \rho_{N}\left(\theta_{B^{\prime}}\right) E_{N}\left(\theta_{B^{\prime}}\right)
$$

$$
\tau\left(\theta, \theta_{B^{\prime}}\right) D^{2} \cos \theta_{B^{\prime}} \sin \theta_{B^{\prime}} d \theta^{\prime} / \pi
$$

where we have used the relations (A3) and (A5). The function $E_{0}(\theta)$ is the average of $E_{0}(\theta, \varphi)$ over $\varphi$ as defined by (A7), and $E_{N}\left(\theta_{B}\right)$ is defined for the baffle points in the same way that $E(\theta)$ was defined for the sphere points. This expression shows that $E(a)$ values averaged over the azimuthal angle will satisfy the integral equation.

The flux striking a small round observation port of radius $p$, centered on the north pole of the sphere, is given by the integral

$$
\int_{0}^{2 \pi}\left[\int_{0}^{\theta_{p}} E(\theta, \varphi) \sin \theta d \theta\right] d \varphi=2 \pi \int_{0}^{\theta_{p}} E(\theta) \sin \theta d \theta
$$

where the upper limit of integration is equal to $\sin ^{-1}$ $(p / R)$. As (All) shows, this integral depends only on the average azimuthal dependence of the source intensity distribution. 